\title{
Improving Stability of Cesium Lead Iodide Perovskite Nanocrystals by Solution Surface Treatments
}

\author{
Dan $\mathrm{Li}^{1}$, Chang-Song Chen ${ }^{1}$, Yi-Hua Wu ${ }^{1,2}$, Zhi-Gang Zhu ${ }^{1,2 *}$, Wan Y. \\ Shih $^{3}$ and Wei-Heng Shih ${ }^{4 *}$
}

1. School of Environmental and Materials Engineering, College of Engineering, Shanghai Polytechnic University, Shanghai 201209, P. R. China

2. Research Center of Resource Recycling Science and Engineering, Shanghai Polytechnic University, Shanghai, 201209, China

3. School of Biomedical Engineering, Science, and Health Systems, Drexel University, Philadelphia, Pennsylvania 19104, United States

4. Department of Materials Science and Engineering, Drexel University, Philadelphia, Pennsylvania 19104, United States

*Corresponding authors:

Prof. Z.-G. Zhu: zhigang_zhu259@163.com, +86-21-5021-5021 (ext. 8325) and Prof. W.-H. Shih: shihwh@drexel.edu, +1-215-8956636

\# Dan Li and Chang-Song Chen contributed equally to this work. 

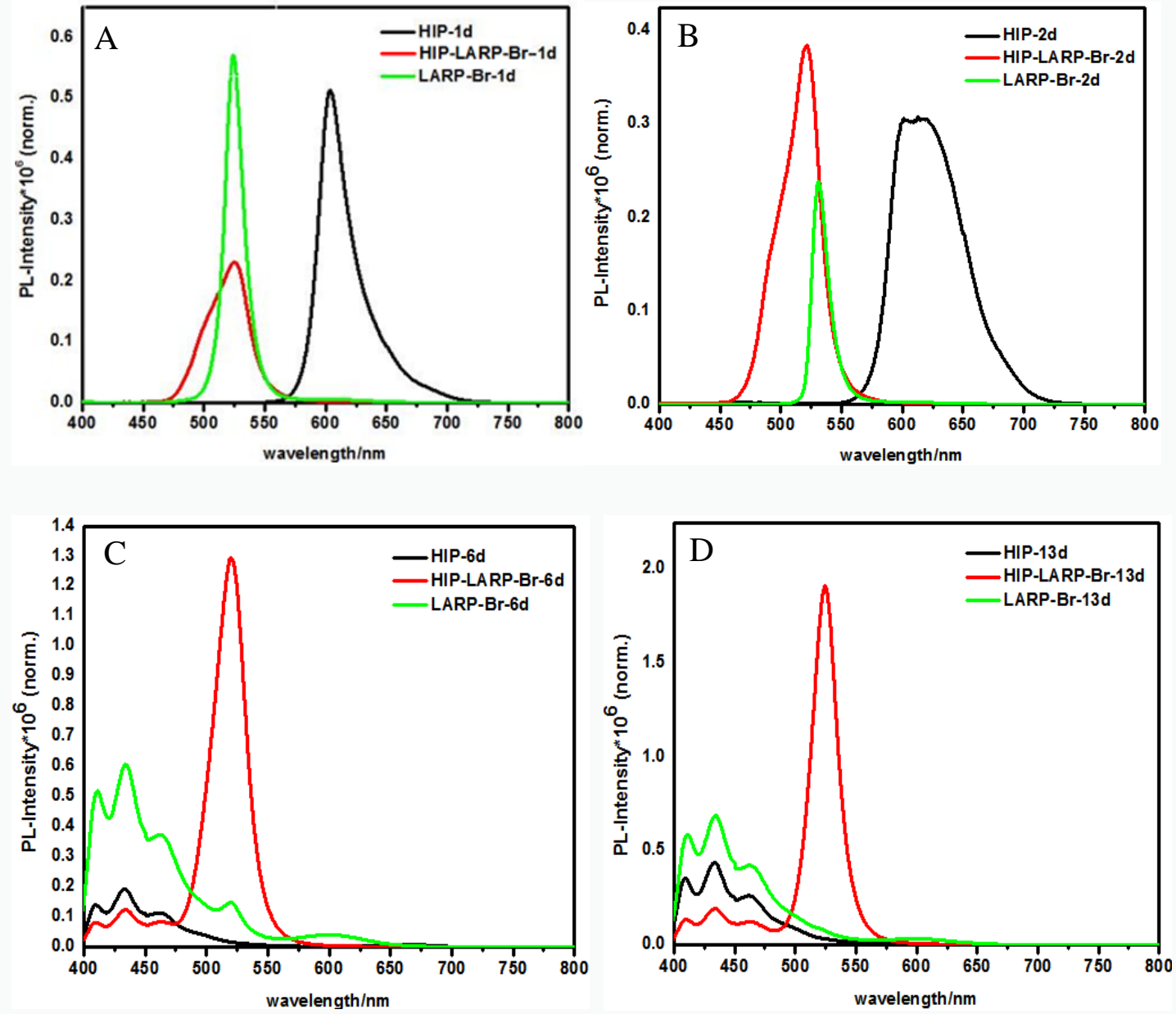

Figure S1. PL spectra of various samples with DMF as the solvent at different days. The black, red, and green lines represent the untreated $\mathrm{CsPbI}_{3}$ sample by HIP method (HIP), the HIP sample treated by $\mathrm{CsPbBr}$ s precursor with the LARP method (HIP-LARP-Br), and the CsPbBr3 sample made by the LARP method (LARP-Br). (A) PL behavior at 1 day (B) 2 days (C) 6 days (D) 13 days. 

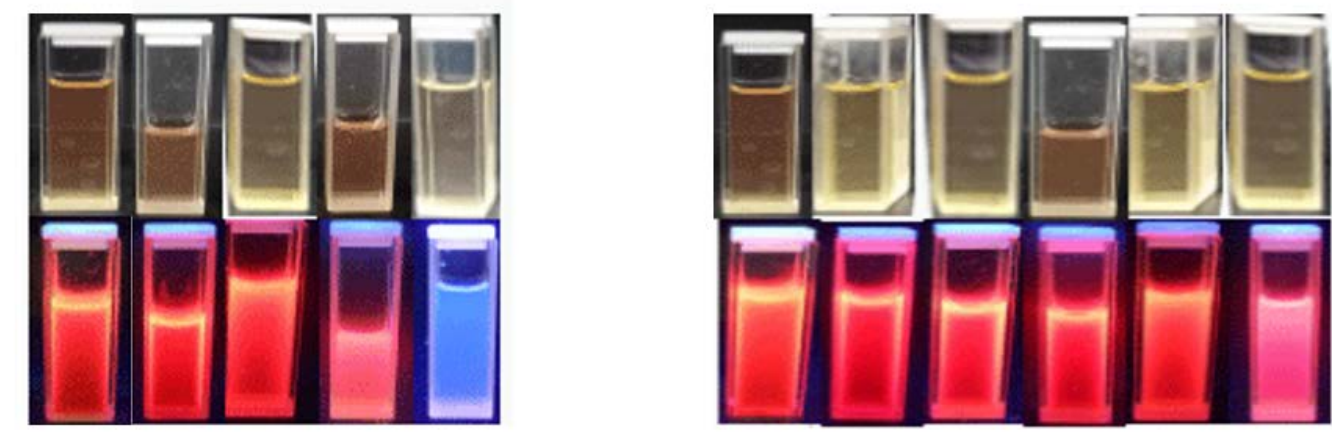

$\begin{array}{lllllllllll}\text { HIP 1d } & 3 d & 5 d & 7 d & 9 d & \text { HIP-LARP-I 1d } & 3 d & 5 d & 7 d & 9 d & 11 d\end{array}$

Figure S2. Images of HIP (left) and HIP-LARP-I (right) under ambient light and UV light as a function of time. 

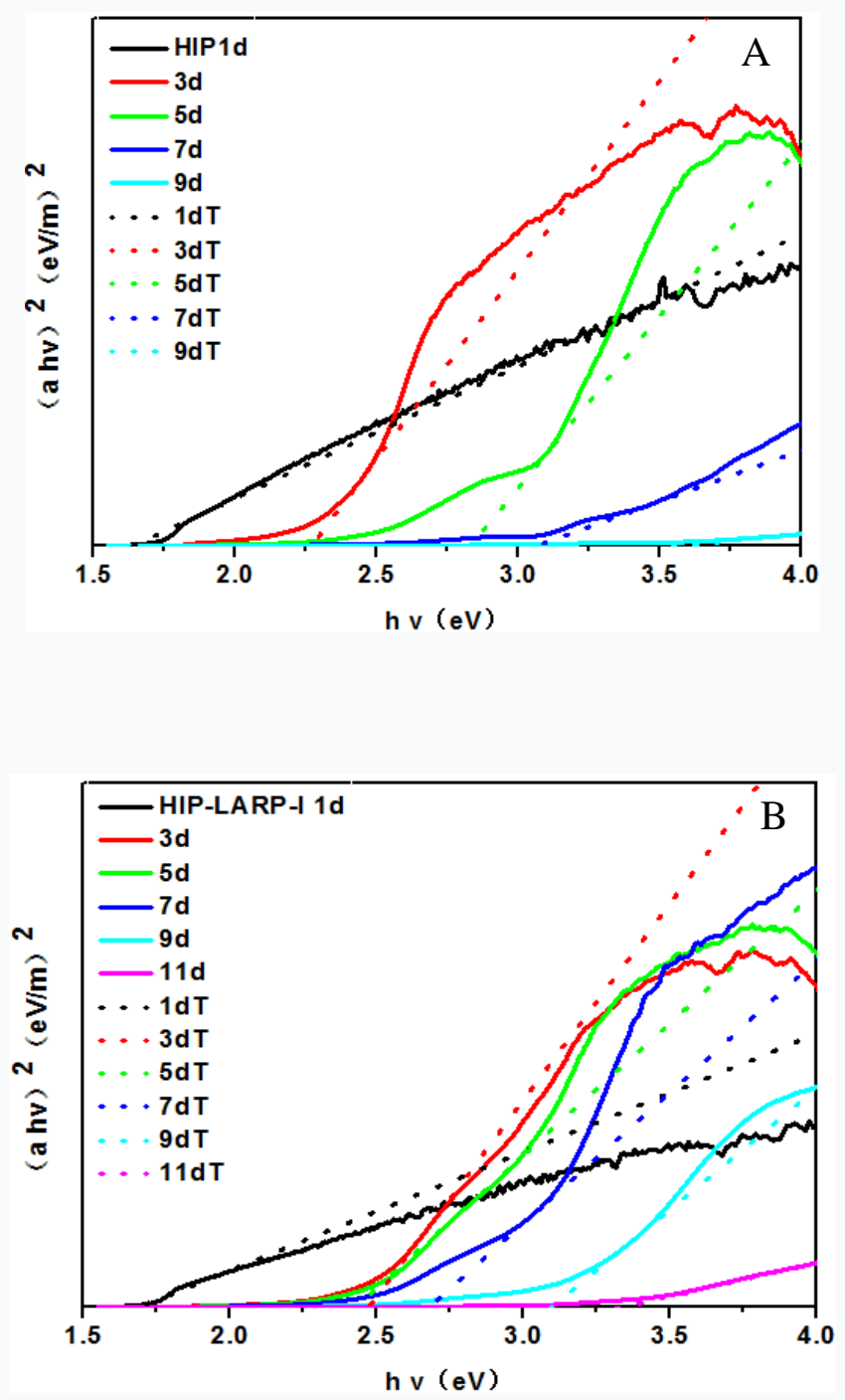

Figure S3. Tauc plots obtained from absorption data of freshly prepared Lead cesium iodide samples with different stability time. 


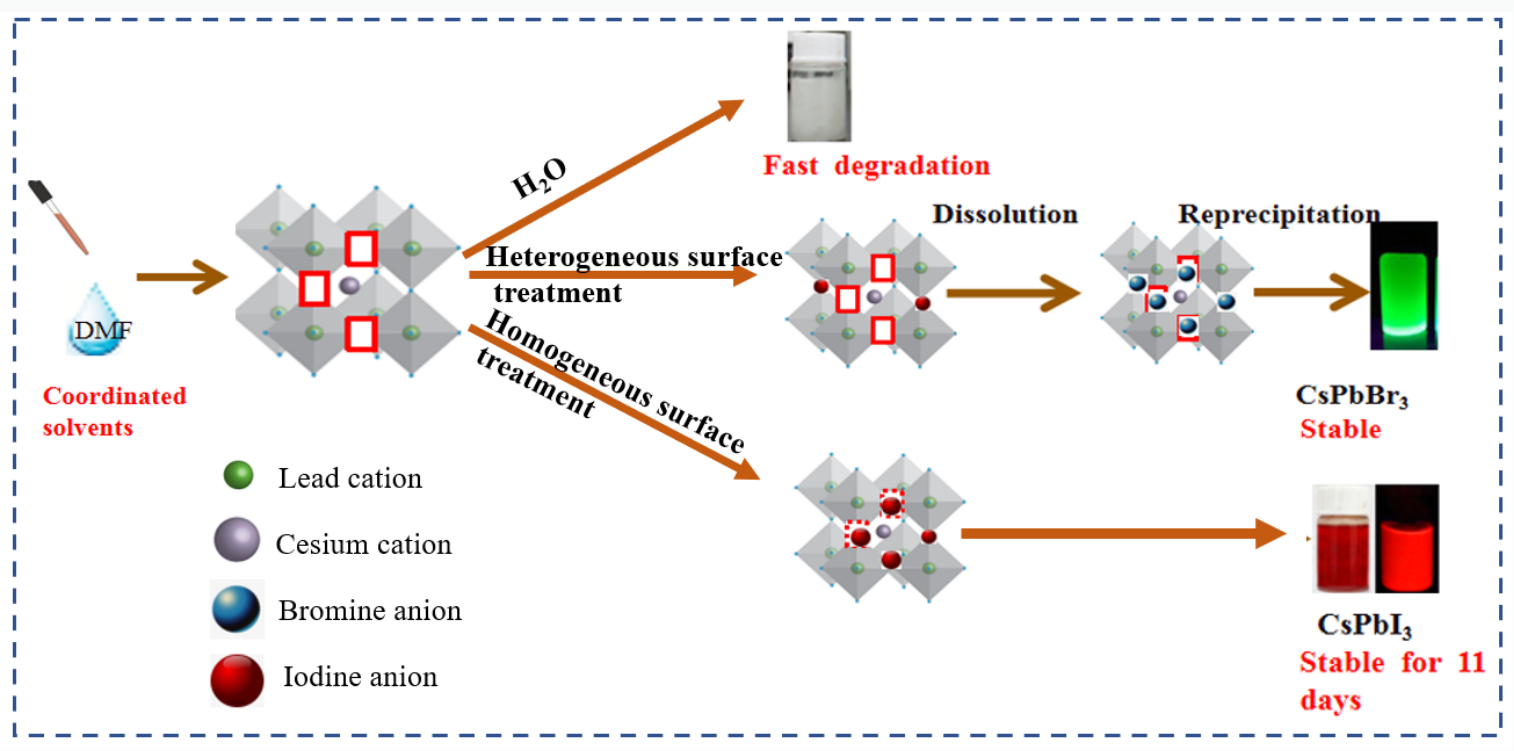

Figure S4. Schematic illustrations of the behaviors of heterogeneous and homogeneous surface treatments. 


\section{Tables}

Table S1. The detailed data of absorbance and absolute quantum yield of cesium lead iodide samples prepared by HIP and HIP-LARP-I methods at different time.

\begin{tabular}{cccc}
\hline samples & PL/nm & Abs & QY/\% \\
\hline HIP1d & 701 & 3.066 & 52.2 \\
HIP-LARP-I 1d & 702 & 2.704 & 48.9 \\
HIP3d & 690 & 3.987 & 67.8 \\
HIP-LARP-I 3d & 687 & 3.955 & 70.2 \\
HIP5d & 686 & 3.646 & 45.5 \\
HIP-LARP-I 5d & 687 & 4.004 & 70.7 \\
HIP7d & 683 & 1.388 & 27.4 \\
HIP-LARP-I 7d & 686 & 3.989 & 69.7 \\
HIP9d & 433 & 0.352 & 0 \\
HIP-LARP-I 9d & 686 & 2.332 & 66 \\
HIP11d (No PL) & - & - & - \\
HIP-LARP-I 11d & 686 & 0.808 & 45 \\
\hline
\end{tabular}


Table S2. The summary table of photoluminescence (PL) peak wavelength, band gap energy obtained from Tauc plot of cesium lead iodide prepared by the HIP and HIP-LARP-I methods at different time.

\begin{tabular}{ccccc}
\hline Samples & Band gap/ev & PL peak/nm & Band gap/nm & correlation value \\
\hline HIP1d & 1.59 & 701 & 779 & 0.46 \\
HIP3d & 1.83 & 690 & 677 & 0.49 \\
HIP5d & 1.98 & 686 & 626 & 0.64 \\
HIP7d & 2.03 & 683 & 611 & 1 \\
HIP9d & 2.04 & 433 & 608 & $/$ \\
\hline Samples & Band gap/ev & PL peak/nm & Band gap/nm & correlation value \\
\hline HIP-LARP-I 1d & 1.57 & 702 & 790 & 0.98 \\
HIP-LARP-I 3d & 1.91 & 687 & 649 & 0.92 \\
HIP-LARP-I 5d & 1.93 & 687 & 642 & (3) \\
HIP-LARP-I 7d & 1.99 & 686 & 623 & $/$ \\
HIP-LARP-I 9d & 2.04 & 686 & 608 & $/$ \\
HIP-LARP-I 11d & 2.06 & 686 & 602 & / \\
\hline
\end{tabular}


Table S3. Summary of recent reports on the use of surface modification to stabilize perovskite

\begin{tabular}{|c|c|c|c|}
\hline Perovskite & Modified ingredients & $\begin{array}{l}\text { Storage } \\
\text { time/condition }\end{array}$ & $\begin{array}{l}\text { Referen } \\
\text { ces }\end{array}$ \\
\hline $\mathrm{CH}_{3} \mathrm{NH}_{3} \mathrm{PbBr}_{3}$ & $\begin{array}{l}\text { APTES } \\
\text { (3-aminopropyl)triethoxysilane) }\end{array}$ & 2.5h (isopropanol) & [41] \\
\hline $\mathrm{CsPbBr}_{3}$ & TDPA (tetradecylphosphonic) & $5 \mathrm{~h}$ (water) & [42] \\
\hline $\mathrm{CsPbBr}_{3}$ & $\begin{array}{l}\mathrm{S}^{2-} \mathrm{DDA}^{+} \text {(didodecyl dimethy-lammonium } \\
\text { sulfide) }\end{array}$ & 34 h (light) & [43] \\
\hline $\mathrm{CsPbBr} 3$ & Mesoporous silica & $100{ }^{\circ} \mathrm{C}$ & {$[32]$} \\
\hline $\mathrm{CsPbBr}_{3}$ & $\mathrm{~S}^{2-} \mathrm{DDA}^{+}$, mesoporous silica, PMMA & $100{ }^{\circ} \mathrm{C}$ & [33] \\
\hline $\mathrm{CsPbBr}_{3}$ & $\begin{array}{l}\text { SEBS } \\
\text { (poly(styrene-ethylene-butylene-styrene) }\end{array}$ & 4 months (water) & [31] \\
\hline $\mathrm{CsPbI}_{3}$ & IDA (2,2'-iminodibenzoic acid) & $\begin{array}{l}15 \text { days (ambient } \\
\text { atmosphere) }\end{array}$ & [44] \\
\hline $\mathrm{CsPbI}_{3}$ & $\begin{array}{l}\text { TMPPA (2,2,4-trimethylpentyl) phosphinic } \\
\text { acid }\end{array}$ & $\begin{array}{l}20 \text { days (ambient } \\
\text { atmosphere) }\end{array}$ & {$[40]$} \\
\hline $\mathrm{CsPbI}_{3}$ & DPPA (diphenyl phosphinic acid) & $\begin{array}{l}6 \text { days (ambient } \\
\text { atmosphere) }\end{array}$ & {$[28]$} \\
\hline $\mathrm{CsPbI}_{3}$ & TOP (trioctylphosphine) & $\begin{array}{l}30 \text { days (ambient } \\
\text { atmosphere) }\end{array}$ & [29] \\
\hline $\mathrm{CsPbI}_{3}$ & $\mathrm{CsPbI}_{3}$ (prepared by LARP method) & $\begin{array}{l}11 \text { days (ambient } \\
\text { atmosphere) }\end{array}$ & $\begin{array}{l}\text { This } \\
\text { work }\end{array}$ \\
\hline
\end{tabular}

